\title{
Comments on gauge-invariance in cosmology
}

\author{
Karim A. Malik ${ }^{1}$ and David R. Matravers ${ }^{2}$ \\ ${ }^{1}$ Astronomy Unit, School of Physics and Astronomy, \\ Queen Mary University of London, Mile End Road, London, E1 4NS, United Kingdom \\ ${ }^{2}$ Institute of Cosmology and Gravitation, University of Portsmouth, \\ Dennis Sciama Building, Portsmouth, PO1 3FX, United Kingdom
}

(Dated: October 31, 2018)

\begin{abstract}
We revisit the gauge issue in cosmological perturbation theory, and highlight its relation to the notion of covariance in general relativity. We also discuss the similarities and differences of the covariant approach in perturbation theory to the Bardeen or metric approach in a non-technical fashion.
\end{abstract}

PACS numbers: 98.80.Jk, 98.80.Cq

arXiv:1206.1478v4

\section{INTRODUCTION}

Perturbation theory is these days a standard tool in theoretical cosmology. Since the introduction of the theory more than sixty years ago, the "gauge issue" has been troubling cosmologists. Although the problem has been resolved by now, at all orders in perturbation theory, there still seems to be some confusion as to its origins. We aim at clarifying these issues in this short note, by highlighting previous results that might be well known to the specialist, but not necessarily to the wider community.

After the pioneering work by Lifshitz in 1946 [1], Bardeen in 1980 2] demonstrated how the gauge issue can be rigorously solved in the metric based approach. The reviews by Kodama and Sasaki [3] and Mukhanov, Feldman and Brandenberger 44 further contributed to the success of this approach. More recently the gauge issue has also been studied beyond linear order in cosmological perturbation theory, leading to a rich body of work using the Bardeen and related gauge-invariant formalisms [5 13.

A different approach has been developed, which is usually referred to as the "covariant" approach, following Ellis and Bruni [14] (and earlier work by [15, 16]). However, as we show below, also the covariant approach has to face the gauge issue if a "background" spacetime is introduced.

But what do we mean by gauge dependence in cosmological perturbation theory, where does it actually come from, and what makes it so difficult? In cosmological perturbation theory we split quantities into a background and small perturbations, both for the matter variables and the metric. Gauge dependence then stems from requiring a unique background spacetime on which the background quantities are defined, that does not follow the coordinate or gauge transformation, whereas the perturbations do obey the transformation. This allows us to talk about e.g. the Friedmann-Robertson-Walker (FRW) background around which we perturb our quantities.

Before we define once more what we mean by gauge- invariance in the setting of cosmological perturbation theory below, let us briefly state what it is not. It should not be mistaken for or mixed up with covariance, the requirement that the governing equations do not depend on the coordinate system chosen. Indeed, it is well known that covariance can be broken in cosmology without conceptual or other problems arising, e.g. in order to choose a particular coordinate system that makes the calculation simpler [17.

Gauge-invariance in cosmological perturbation theory should also not be confused with the gauge choice in standard General Relativity (GR). We can choose four arbitrary coordinate functions in the metric tensor (thanks to the four constraint equations in Einstein's field equations, see e.g. Ref. [18]). In cosmological perturbation theory the symmetries of the physical spacetime, namely homogeneity and isotropy, lead us to choose a background that is FRW (with zero background curvature for convenience), and we can then use covariance to choose our coordinates in the background such that the line element has a particularly simple form, i.e.

$$
d s^{2}=d t^{2}+a^{2}(t) \delta_{i j} d x^{i} d x^{j},
$$

where $a$ is the scale factor and $\delta_{i j}$ the flat background metric. This is related to the "geodesic slicing" in numerical relativity, which is selected by choosing a lapse function normalised to unity, and choosing a vanishing shift vector selects Gaussian normal coordinates (see e.g. Ref. [19]). Hence the "standard GR coordinate freedom" is used, actually used up, in choosing and specifying the background.

This note is aimed at the non-specialist and we try to keep our discussions as non-technical as possible, working rather by way of example than by introducing theorems. Nevertheless, in the following two sections we have to define gauge-dependence and gauge-invariance in a more rigorous way than above. In Section IVA we show how quantities in the covariant formalism are related to the ones in standard metric perturbation theory, and comment briefly on the issue of non-locality in perturbation theory in Section IVB, and conclude with a short discussion in Section V Finally we highlight in Appendix A 


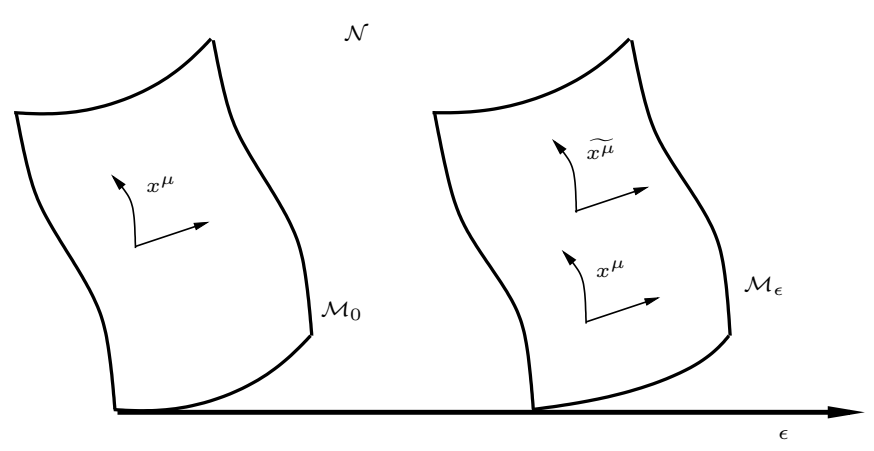

FIG. 1: The manifolds $\mathcal{M}_{0}$ and $\mathcal{M}_{\epsilon}$, embedded in the higher dimensional manifold $\mathcal{N}$. The small parameter $\epsilon$ is an additional dimension (or label) in $\mathcal{N}$.

the difference between covariance and gauge-invariance.

We use predominantly coordinate time $t$, and denote derivatives with respect to $t$ with a dot. Conformal time $\eta$ is related to $t$ by $d t=a d \eta$, where $a$ is the scale factor. Greek indices $\mu, \nu$, and $\lambda$ range from 0 to 3, lower case Latin indices, $i, j$, and $k$, have the range $1,2,3$.

\section{THE ORIGIN OF GAUGE DEPENDENCY IN PERTURBATION THEORY}

So far we have mentioned that there is a problem in cosmological perturbation theory often referred to as the "gauge issue", but in the previous section we only sketched it very roughly. Let us therefore now turn to the origin of gauge dependence and define what we mean by gauge, gauge dependence, and gauge invariance. We shall use the FRW background universe as an example spacetime. As stated above, we shall attempt to be as non-technical as possible in this note, and refer the interested reader to the seminal papers by Stewart and Walker 20] and Stewart 21] for technical details, and to Ref. 11] whose notation we follow where possible.

The physical domain ("the universe") in which we work in cosmology can be regarded as a single manifold $\mathcal{M}_{\epsilon}$. In perturbation theory, following Ref. [20] we introduce another, fiducial, manifold $\mathcal{M}_{0}$ (frequently referred to as the "background" manifold). A correspondence or map (one-to-one mapping or diffeomorphism) between $\mathcal{M}_{\epsilon}$ and $\mathcal{M}_{0}$ is referred to as a gauge and the correspondence is generated by a vector field, the gauge generator.

As illustrated in Fig. 1 the manifolds $\mathcal{M}_{\epsilon}$ and $\mathcal{M}_{0}$ are treated as being embedded in the higher dimensional manifold $\mathcal{N}=\mathcal{M} \times \mathcal{R}$. The parameter $\epsilon$ allows us to distinguish between the manifolds $\mathcal{M}_{0}$ and $\mathcal{M}_{\epsilon}$, the former, the background, being at label $\epsilon=0$. We can also introduce coordinate systems. We start out with $\left\{x^{\mu}\right\}$ on $\mathcal{M}_{0}$, and identifying the points on $\mathcal{M}_{\epsilon}$ with those on $\mathcal{M}_{0}$ we can also label the points on $\mathcal{M}_{\epsilon}$ with the coordinates $\left\{x^{\mu}\right\}$. Then relabelling the points on $\mathcal{M}_{\epsilon}$ induces a gauge transformation.

The gauge issue arises from splitting quantities, defined on $\mathcal{N}$ into a background part, on $\mathcal{M}_{0}$, which usually depends on fewer dimensions (or coordinates) than the physical part (on $\mathcal{M}_{\epsilon}$ ) which defines the perturbation which usually depends on the full set of dimensions (coordinates) of the spacetime. When we change the coordinate system on the physical space $\mathcal{M}_{\epsilon}$ by a small amount $x^{\mu} \rightarrow \tilde{x^{\mu}}$ and require that the coordinates of the background remain unperturbed, to keep a unique background spacetime (e.g. the FRW background in standard cosmology) the perturbed quantities on $\mathcal{M}_{\epsilon}$ will undergo a gauge transformation generated by this relabelling of coordinates.

Let us rephrase the above in a slightly more technical form. We can split any tensorial quantity $\mathcal{Q}$ into a background part, $\mathcal{Q}_{0}$ and a small perturbation $\delta \mathcal{Q}$, that is

$$
\mathcal{Q} \equiv \mathcal{Q}_{0}+\delta \mathcal{Q}
$$

Note that the quantity $\mathcal{Q}$ is defined on physical manifold $\mathcal{M}_{\epsilon}$ ("background+ perturbation"), whereas $\mathcal{Q}_{0}$ is defined on $\mathcal{M}_{0}$ ("background", with $\epsilon=0$ ).

We can now change the coordinate system $x^{\mu}$ on $\mathcal{M}_{\epsilon}$ to a new system $\tilde{x}^{\mu}$ which is related to the old one by a small amount, $\delta x^{\mu}$ that is

$$
\tilde{x}^{\mu}=x^{\mu}+\delta x^{\mu} .
$$

This change will also induce ${ }^{1}$ (in general) a change in the perturbation $\delta \mathcal{Q}$, that is

$$
\delta \mathcal{Q}(x) \rightarrow \widetilde{\delta \mathcal{Q}}(\tilde{x}),
$$

however, the background quantity remains unchanged,

$$
\mathcal{Q}_{0}(x)=\widetilde{\mathcal{Q}_{0}}(\tilde{x}) .
$$

Studying the transformation behaviour of the perturbed quantities allows us to "fix" or correct this different and disparate behaviour of the quantities (i.e. the background quantities remain fixed, the perturbations are allowed to vary), and construct gauge-invariant quantities (free of gauge-artefacts, the $\delta x^{\mu}$ defined in Eq. (2.2). Note that $O(\delta \mathcal{Q})=O\left(\delta x^{\mu}\right)=O(\epsilon)$.

The terms "gauge" and "gauge invariance" are used in different ways in the literature, we mentioned the use of the term in GR in the previous section. Another definition of gauge invariance, due to Stewart and Walker,

\footnotetext{
1 We are choosing here the "passive" point of view, to calculate the change of the variables under a gauge transformation. In this approach, one calculates the effect that a change of coordinate system, Eq. 2.2, has on the variables. In the active approach the change in the variables is calculated directly from the "action" of the gauge generator $\delta x^{\mu}$ (see Ref. 11 for details).
} 
is sometimes also referred to as identification gauge invariance ("i.g.i." in Stewart and Walker [20]) or "strong" gauge invariance. For a perturbed quantity to be identification gauge invariant its background part has to be either, vanishing ${ }^{2}$, or constant, or constructed out of linear combination of Kronecker deltas 20. This is usually referred to as the "Stewart-Walker Lemma". This Lemma is rather restrictive, it doesn't allow for any gauge-artefacts (the gauge generators $\delta x^{\mu}$ ) to appear in the transformation equations for the perturbed variables.

The definition of gauge invariance we use here, following Bardeen, is weaker than the identification gauge invariance: we require that there are no gauge artefacts (the "gauge generators", or $\delta x^{\mu}$ defined in Eq. 2.2p) left in the governing equations, if they are subjected to the transformation 2.2 . Similarly, a gauge-independent quantity is one that doesn't "pick up" gauge artefacts if subjected to the transformation Eq. (2.2). Hence quantities can be gauge-invariant, in this more general sense, but not satisfy the Stewart-walker lemma. We will illustrate this with an example in the following section.

\section{METRIC OR COORDINATE BASED PERTURBATION THEORY}

In Section II we discussed the behaviour of tensorial quantities under a gauge transformation in very general terms. We now derive how perturbations transform in a particular spacetime, using the transformation behaviour of the first order or linear density perturbation in a FRW background, where the background momentum density vanishes, as an example ${ }^{3}$, and then discuss how to construct gauge-invariant quantities. The derivation follows as closely as possible Ref. [11.

Under the transformation Eq. 2.2 we get, using the passive approach, that the energy density in the new ("tilde") system is related to the density in the old system as

$$
\begin{aligned}
\tilde{\rho}\left(\tilde{x}^{\mu}\right) & =\tilde{\rho}\left(x^{\mu}+\delta x^{\mu}\right) \\
& =\tilde{\rho}\left(x^{\mu}\right)+\tilde{\rho}_{, \mu} \delta x^{\mu},
\end{aligned}
$$

where we used a Taylor expansion, truncating here and in the following at linear order, in the second line. Then

\footnotetext{
2 Either the background part has to be zero, or its Lie derivative with respect to the gauge-generator has to be zero, i.e. $\mathrm{Ł}_{\delta x^{\mu}} \mathcal{Q}_{0}=$ 0 , to be precise [20. The restrictive notion of i.g.i. can be extended to order $n$ in perturbation theory by requiring that $\mathrm{Ł}_{\delta x^{\mu}} \mathcal{Q}_{m}=0$, where $m$ is in the range of $0 \ldots n-1[5]$. Note that the Lie derivative has to act on the whole tensorial quantity, and not just a particular component of it.

3 We use the energy density here as an example for the transformation behaviour of a scalar quantity. In more complex spacetimes than FRW, the energy density has to be derived from the energymomentum tensor specifically.
}

using the expansion Eq. 2.1) for the energy density, and also applying Eq. 2.4 to the energy density, that is we require $\tilde{\rho}_{0}(t)=\rho_{0}(t)$ (the FRW background is time dependent only) we arrive at the standard result

$$
\widetilde{\delta \rho}=\delta \rho-\dot{\rho}_{0} \delta t
$$

the transformation of the energy density under a gauge transformation, at linear order. Here we used the standard $3+1$ split for the gauge generator $\delta x^{\mu}$, that is $\delta x^{\mu} \equiv\left[\delta t, \delta x^{i}\right]$.

From the transformation of $\rho$, Eq. (3.2), we can also see how the Stewart-Walker lemma "works". If the quantity in the background is zero or constant, the second term in Eq. (3.2) would also be zero, and hence the perturbation in the new and the old coordinate system would be identical, that is gauge-invariant in the strong sense.

The perturbed FRW metric for scalar perturbations and a flat background is given by

$$
\begin{aligned}
d s^{2}= & -(1+2 \phi) d t^{2}+2 a B_{, i} d t d x^{i} \\
& +a^{2}\left[(1-2 \psi) \delta_{i j}+E_{, i j}\right] d x^{i} d x^{j} .
\end{aligned}
$$

Here $\phi$ is the lapse function, $B$ and $E$ describe the shear, and $\psi$ is the curvature perturbation related to the perturbed intrinsic curvature of spatial 3-hypersurfaces by ${ }^{(3)} R=4 \nabla^{2} \psi / a^{2}$ (see e.g. Ref. [12]).

To keep the algebra in this note at a minimum we do not show here how to derive and calculate the transformation behaviour of the metric tensor and refer the interested reader to Refs. 11 and 12 for details. We simply quote the result for e.g. the curvature perturbation here,

$$
\widetilde{\psi}=\psi+H \delta t
$$

where $H \equiv \frac{\dot{a}}{a}$.

We now have two perturbed quantities and their transformations at our disposal, and this allows us to construct a gauge-invariant quantities as follows. Equations 3.2 and 3.4 let us construct two related gauge-invariant quantities, since we can decide to choose two particular gauges or hypersurfaces: working on uniform density hypersurfaces, defined as $\widetilde{\delta \rho}=0$, we get from Eq. 3.2 for the temporal gauge shift $\delta t$,

$$
\left.\delta t\right|_{\widetilde{\delta \rho=0}}=\frac{\delta \rho}{\dot{\rho}_{0}} .
$$

Substituting this expression into Eq. (3.4) we get

$$
\left.\psi\right|_{\widetilde{\delta \rho=0}}=\psi+\frac{H}{\dot{\rho}_{0}} \delta \rho
$$

the curvature perturbation on uniform density hypersurfaces.

It can be shown, that this quantity is conserved on very large scales for adiabatic systems that is with barotropic 
equation of state (independent of the underlying theory of gravity) 22], and is usually denoted by $\zeta$, where for historic reasons $-\zeta=\left.\psi\right|_{\widetilde{\delta \rho}=0}$.

Similarly, instead of working on uniform density hypersurfaces, we can choose uniform curvature hypersurfaces, defined as $\widetilde{\psi}=0$, which gives from Eq. 3.4

$$
\left.\delta t\right|_{\widetilde{\psi}=0}=-\frac{\psi}{H} .
$$

Substituting this expression into Eq. (3.2) we get

$$
\left.\delta \rho\right|_{\widetilde{\psi}=0}=\delta \rho+\frac{\dot{\rho}_{0}}{H} \psi
$$

the density perturbation on uniform curvature hypersurfaces.

Both quantities defined in Eqs. (3.6) and 3.8 above are gauge-invariant, that is invariant under a transformation of the form Eq. (2.2). This can be readily seen from the respective definitions, as in both cases the gauge-artefacts $\delta t$, that the "raw" quantities would pick up, cancel in the respective combinations. It is this definition of gaugeinvariance, namely that the gauge-invariant quantities do not pick up gauge-artefacts $\delta x^{\mu}$, that we use and which is, arguably, more popular these days ${ }^{4}$. This notion of gauge-invariance can also be readily extended to higher order (see e.g. Refs. 11, 12 for examples at second order, and Ref. 23] for examples at third order).

\section{THE COVARIANT APPROACH TO PERTURBATION THEORY}

We now turn to the "covariant approach" to cosmological perturbation theory and how it deals with any potentially ensuing gauge issues. One might be tempted, given the approach's name, that here the gauge issue is avoided altogether, after all GR doesn't suffer from the perturbation theory gauge issue besides the "normal" coordinate freedom, as pointed out in Section 1 .

The covariant approach to perturbation theory [14-16. $24-27$ allows in principle to avoid the gauge issue, as it does not require to specify the metric from the beginning of the calculation. However, as soon as one imposes a split into a particular background and perturbations, the same issues as described above in Section II and troubling the metric approach will "haunt" the covariant approach.

One possibility to remove gauge artefacts is to use the Stewart-Walker lemma, and use quantities that vanish in

\footnotetext{
4 Note that neither the "raw" quantities nor their gauge-invariant combinations satisfy the Stewart-Walker lemma: the energy density has in general a non-zero background part, and the curvature perturbation is related to the expansion and hence the Hubble parameter in the background.
}

the background. Hence in FRW we can construct the spatial gradient of a scalar, and since the background is time dependent only, this has no correspondence in the background (i.e. removes gauge terms, see below). However, this only works at linear order in a standard FRW background: if we think perturbatively again, as the second order quantities "live" in the first order time and space dependent background, which does allow gradients. This is also the reason why second order tensor perturbations are no longer gauge-invariant, as they have first order tensor perturbations acting as a background 11 .

\section{A. Relating "covariant" and "Bardeen" quantities:}

We can now relate quantities in the covariant formalism and in the Bardeen formalism (see e.g. Refs. 24] and 26] for earlier work on this topic). In the covariant formalism quantities (gradients) are projected down onto spatial three sections using the projection tensor defined below, Eq. 4.3), relative to some vector field, usually chosen to be the fluid four-velocity. This suggests that the quantities constructed in this way are closely related to quantities in the Bardeen formalism in comoving gauge. That is indeed the case, as we show below.

In the following we are using the definitions from Ref. 25, and only consider scalar quantities. We choose here a particular quantity for comparison, though this is without loss of generality. The "comoving fractional density gradient" is defined in Ref. 25] as

$$
\mathcal{X}_{\mu} \equiv \frac{a}{\rho}^{(3)} \nabla_{\mu} \rho
$$

and

$$
{ }^{(3)} \nabla_{\mu} \rho \equiv h_{\mu}^{\lambda} \nabla_{\lambda} \rho,
$$

with the projection tensor

$$
h_{\mu \nu} \equiv g_{\mu \nu}+u_{\mu} u_{\nu}
$$

and $u_{\mu}$ is a physically defined four-velocity which reduces in FRW to the that of the "fundamental observers" $\left(\nabla_{\mu}\right.$ is the standard covariant derivative, which here however reduces to the partial derivative because it is applied to a scalar quantity).

In order to relate the two formalisms, we now substitute the Bardeen formalism (first order) quantities into the above. We have for the components of the fourvelocity $u_{\mu}$ (see e.g. Ref. [12]) and using conformal time $\eta$

$$
\begin{aligned}
& u_{\mu}=a\left[-(1+\phi), \partial_{i}(v+B)\right], \\
& u^{\mu}=a^{-1}\left[(1-\phi), \partial^{i} v\right],
\end{aligned}
$$

where is $B$ the shift function given in Eq. (3.3), and $v$ the scalar velocity perturbation, and get for the density gradient ${ }^{(3)} \nabla_{\mu} \rho$, to first order

$$
{ }^{(3)} \nabla_{i} \rho=\partial_{i} \delta \rho+\rho_{0}^{\prime} \partial_{i}(v+B),
$$


the temporal part being identically zero. The right-handside of Eq. 4.5 agrees, modulo the gradient, with the expression given in Ref. [12] for the comoving density perturbation, namely

$$
\widetilde{\delta} \rho_{\text {com }} \equiv \delta \rho+\rho_{0}^{\prime}(v+B),
$$

which is what we expected.

\section{B. The issue of non-locality}

We finally reflect on another topic of frequent discussions with colleagues, which was also discussed recently in Refs. 28, namely the issue of whether perturbation theory is "local" or "non-local", with regard to metric and covariant approaches.

The question here is, how to deal with gradient terms that appear in the governing equations. Gradient terms are already present in the definitions of the perturbed variables, e.g. in the definition of the 4-velocity, Eq. (4.4) above, or in the spatial section of the metric tensor, if we decompose the variables on spatial 3 -hypersurfaces. This decomposition leads to the standard "nomenclature" for matter and metric perturbations, labelling them by their transformation behaviour on the spatial 3-hypersurfaces: we can decompose 3-vectors into a scalar (the curlfree part) and a divergence-free vector, and similarly 3tensors into scalars, vectors (divergence-free), and tensors (divergence-free and traceless). This decomposition is popular in cosmological perturbation theory, as at linear order the governing equations for the different types of perturbations decouple 2, which simplifies the calculations.

To get rid of the gradients in the ensuing equations, we have to formally integrate the equations, or if we work in Fourier space, solve the governing equations for the mode functions (see e.g. Refs. [4, 29]). This is straight forward at linear order, where only simple operators of the form $\partial_{i}$ (acting on the whole equation), or $\partial_{i} \partial^{i}$ arise.

However beyond linear order this is no longer the case, and to rewrite the governing equations in closed form in terms of scalar quantities we will inevitable pick up inverse gradient terms (see e.g. Refs. 9, 30, 31]). It is these inverse gradients terms that are usually referred to as "non-local". This also means that in Fourier space this will lead to mode-mixing, which requires solving convolution integrals.

There are several ways to deal with this issue: a) We can use a large scale or super-horizon approximation and a gradient expansion, that is simply neglect the gradient terms at a particular order (see e.g. Refs. [30, 32, 33]).

b) Alternatively, we can keep the gradient terms as they are without solving for the scalars, as is common in "standard GR" and the covariant formalism, as advocated in e.g. Refs. 14, 28 (at linear and higher order, respectively). But then we can only calculate e.g. the power spectrum of the gradient of the density perturbation. To get the power spectrum of the density perturbation itself, we will have to invert the equations to get rid of the gradients.

We could also in the Bardeen formalism keep our equations "local", if we do not invert the gradient terms. But then we would only have governing equations for the gradients of scalars as in the covariant formalism, and have the same problems as mentioned above.

c) Or we can simply suffer the consequences of deriving governing equations for the scalar variables at higher order, namely the introduction of non-local terms, and very complex equations. However, although complex the ensuing equations can be readily solved numerically (see e.g. Refs. [31, 34]).

To conclude this section: if we want governing equations for purely scalar quantities, both the covariant and the Bardeen formalism will lead to equations with inverse gradient or non-local terms.

\section{CONCLUSION}

As GR is a non-linear theory, we usually have to resort to some form of approximative scheme to solve the governing equations. Perturbation theory allows us to solve the equations iteratively order by order.

In cosmological perturbation theory we have to allow for perturbations of spacetime itself, by using a perturbed metric tensor and correspondingly perturbing the coordinates. This is a major difference to classical perturbation theory in e.g. fluid dynamics, which uses a fixed Euclidean or Minkowsky spacetime.

Gauge dependence in cosmological perturbation theory comes from the need to work with a unique background spacetime, that remains fixed under small coordinate transformations. We can begin with the physical spacetime $\mathcal{M}_{\epsilon}$ (see Fig. 1), on which we define all unperturbed variables. We then start our perturbative expansion, by introducing a fiducial background spacetime $\mathcal{M}_{0}$ on which we define the background quantities, both matter and metric variables. The perturbations are then defined as the difference between the quantities defined on the physical spacetime $\mathcal{M}_{\epsilon}$ and the unperturbed quantities on $\mathcal{M}_{0}$.

We relate the background quantity to its perturbed version (on $\mathcal{M}_{\epsilon}$ ), i.e. get a correspondence between the perturbed and unperturbed realisations of the quantities using a one-to-one map, parametrised by a vector field, the gauge generator. A gauge transformation changes this correspondence and hence the perturbations unless they are gauge-invariant. We can always construct gauge-invariant variables, at any order, by studying their transformation behaviour, and then combining the variables in such a way that the gauge artefacts that they pick up in the transformation cancel. Note that a coordinate transformation transforms all the 
coordinates, i.e. in both the background and physical spacetimes, whereas a gauge transformation does not affect the background quantities.

The gauge issue outlined in this note will arise in any theory that keeps the background fixed under small coordinate transformations. Once we have decided to use cosmological perturbation theory, a choice of gauge is therefore always required if such a theory is used, even when dealing with physically measurable quantities and working on small scales. Hence dealing with the gauge issue is not optional or a question of taste, it is dictated by the fact that we have chosen cosmological perturbation theory and a unique background spacetime. Of course, for many applications Newtonian theory will be sufficient, and hence no gauge issue will arise.

Moreover, we should consider the gauge freedom as an opportunity to choose the simplest form of the governing equations possible, not as a problem. As long as we use the formalism with care, namely make sure that no gauge artefacts or "unphysical degrees of freedom" are left in the equations, there will be no ambiguity when we relate observations with theory.

\section{Acknowledgments}

The authors are grateful to Ellie Nalson, Iain Brown, Adam Christopherson, Juan Valiente-Kroon, and David Wands for useful discussions and comments. KAM is supported in part by the STFC under grants ST/G002150/1, ST/H002855/1, and ST/J001546/1.

\section{Appendix A: Covariance versus Gauge-invariance by example}

In this appendix we present a very simple example to highlight the difference between covariance or invariance to general coordinate transformations, and gaugeinvariance as used in cosmological perturbation theory, as defined in Section II

To keep things simple, let us study a scalar function dependent on a single coordinate only, $\rho=\rho(t)$. A scalar is by definition invariant under coordinate transformation

$$
\rho(t)=\widetilde{\rho}(\tilde{t}),
$$

where $\rho$ is the function evaluated in the coordinate system $x^{\mu}$ and $\widetilde{\rho}$, the function evaluated in coordinate system $\tilde{x}^{\mu}$ (note that $t \equiv x^{0}$ ).

To see the effect of the different types of transformation, let's assume we have the following simple dependence on $t$ for our function,

$$
\rho(t) \equiv t+\epsilon t
$$

where for now we make no assumption about $\epsilon=$ const, i.e. we here do not assume that $\epsilon$ is small.
We now introduce a new coordinate system $\tilde{x}$, related to the original system $x$ by

$$
\tilde{t}=t+\delta t
$$

where again, we make no assumption about the size $\delta t$. From Eq. A1 and A3 we then get

$$
\begin{aligned}
\rho(t) & =\rho(\tilde{t}-\delta t) \\
& =\tilde{t}-\delta t+\epsilon(\tilde{t}-\delta t)=\tilde{\rho}(\tilde{t}),
\end{aligned}
$$

where the last line gives the functional dependence of $\widetilde{\rho}$ on $\tilde{t}$.

\section{1. "Covariance at work"}

Let us first show the effect of a covariant transformation (no need to properly perturb or expand things). We can now evaluate the function $\rho$ at a particular point $Q$, with coordinate $t=t_{\mathrm{c}}$, say, and find that

$$
\rho\left(t=t_{\mathrm{c}}\right)=t_{\mathrm{c}}+\epsilon t_{\mathrm{c}},
$$

rather unsurprisingly. The coordinate value in $\tilde{x}$ for $\mathrm{Q}$ is $\tilde{t}=t_{\mathrm{c}}+\delta t$, and substituting into $\widetilde{\rho}(\tilde{t})$ we get

$$
\tilde{\rho}\left(\tilde{t}=t_{\mathrm{c}}+\delta t\right)=t_{\mathrm{c}}+\epsilon t_{\mathrm{c}},
$$

that is the same functional value as in the system $x$, as required.

\section{2. "Gauge-dependence at work"}

To see the effect of the gauge transformation, we have to perturb and expand our quantities, so let's assume $\epsilon \ll 1$ and $\delta t=O(\epsilon)$. From Eq. (A2) we can immediately read off the background or zeroth order part and the perturbation of $\rho$, namely

$$
\rho_{0}(t)=t, \quad \delta \rho(t)=\epsilon t .
$$

The crucial difference to the covariant case above is that now we require for the background part of the variable, using Eq. (2.4,

$$
\rho_{0}(t)=\widetilde{\rho}_{0}(t),
$$

that is the functional dependence of the background functions $\rho_{0}$ and $\widetilde{\rho}_{0}$ on the independent variable is the same (i.e. in cosmology we would want the same background FRW spacetime).

Evaluating our variables in the system $\tilde{x}$ we then have

$$
\rho_{0}(t)=\widetilde{\rho}_{0}(\tilde{t})=\tilde{t},
$$

for the background part, and

$$
\begin{aligned}
\delta \rho(t) & =\epsilon t=\epsilon(\tilde{t}-\delta t) \\
& =\epsilon \tilde{t}+O\left(\epsilon^{2}\right) .
\end{aligned}
$$


for the perturbation.

Again evaluating at the point $Q$, we have in $x$ that $\rho(Q)=t_{\mathrm{c}}(1+\epsilon)$, but in $\tilde{x}$ we have (to linear order) $\widetilde{\rho}(Q)=t_{\mathrm{c}}(1+\epsilon)+\delta t$, so there is discrepancy.

The standard result how the perturbation $\delta \rho$ changes under a gauge transformation $t \rightarrow t+\delta t$ was given above in Eq. (3.2) (repeated here for convenience)

$$
\widetilde{\delta \rho}=\delta \rho-\dot{\rho}_{0} \delta t
$$

and we see that this fixes the discrepancy described above.
[1] E. Lifshitz, J. Phys. (USSR) 10, 116 (1946).

[2] J. M. Bardeen, Phys. Rev. D 22, 1882 (1980).

[3] H. Kodama and M. Sasaki, Prog. Theor. Phys. Suppl. 78, 1 (1984).

[4] V. F. Mukhanov, H. A. Feldman and R. H. Brandenberger, Phys. Rept. 215, 203 (1992).

[5] M. Bruni, S. Matarrese, S. Mollerach and S. Sonego, Class. Quant. Grav. 14, 2585 (1997) arXiv:grqc/9609040.

[6] V. F. Mukhanov, L. R. W. Abramo and R. H. Brandenberger, Phys. Rev. Lett. 78, 1624 (1997) arXiv:grqc/9609026.

[7] K. A. Malik and D. Wands, Class. Quant. Grav. 21, L65 (2004) arXiv:astro-ph/0307055.

[8] H. Noh and J. c. Hwang, Phys. Rev. D 69, 104011 (2004).

[9] N. Bartolo, E. Komatsu, S. Matarrese and A. Riotto, Phys. Rept. 402, 103 (2004) arXiv:astro-ph/0406398.

[10] K. Nakamura, Prog. Theor. Phys. 117, 17 (2007) arXiv:gr-qc/0605108.

[11] K. A. Malik and D. R. Matravers, Class. Quant. Grav. 25, 193001 (2008) arXiv:0804.3276 [astro-ph]].

[12] K. A. Malik and D. Wands, Phys. Rept. 475, 1 (2009) arXiv:0809.4944 [astro-ph]].

[13] A. J. Christopherson, K. A. Malik, D. R. Matravers and K. Nakamura, Class. Quant. Grav. 28, 225024 (2011) arXiv:1101.3525 [astro-ph.CO]].

[14] G. F. R. Ellis and M. Bruni, Phys. Rev. D 40, 1804 (1989).

[15] R. K. Sachs, in Relativity, Groups and Topology, ed C. De Witt and B. De Witt, Gordon Breach, New York (1964).

[16] S. W. Hawking, Astrophys. J. 145, 544 (1966).

[17] G. F. R. Ellis and D. R. Matravers GRG Volume 27, Number 7, 777 (1995).

[18] C. W. Misner, K. S. Thorne and J. A. Wheeler, Gravita- tion, San Francisco 1973, 1279p

[19] T. W. Baumgarte and S. L. Shapiro, Numerical Relativity, CUP (2010)

[20] J. M. Stewart and M. Walker, Proc. Roy. Soc. Lond. A 341, 49 (1974).

[21] J. M. Stewart, Class. Quant. Grav. 7, 1169 (1990).

[22] D. Wands, K. A. Malik, D. H. Lyth and A. R. Liddle, Phys. Rev. D 62, 043527 (2000) arXiv:astro$\mathrm{ph} / 0003278$.

[23] A. J. Christopherson and K. A. Malik, JCAP 0911, 012 (2009) arXiv:0909.0942 [astro-ph.CO]].

[24] M. Bruni, P. K. S. Dunsby and G. F. R. Ellis, Astrophys. J. 395, 34 (1992).

[25] A. Challinor, A. Lasenby, Phys. Rev. D58, 023001 (1998), astro-ph/9804150.

[26] D. Langlois and F. Vernizzi, JCAP 0702, 017 (2007) astro-ph/0610064.

[27] C. G. Tsagas, A. Challinor and R. Maartens, Phys. Rept. 465, 61 (2008) arXiv:0705.4397 [astro-ph]].

[28] C. Clarkson and B. Osano, Class. Quant. Grav. 28, 225002 (2011) [Erratum-ibid. 29, 079601 (2012)] arXiv:1102.4265 [gr-qc]]; C. Clarkson, arXiv:1108.4513 astro-ph.CO]].

[29] K. Nakamura, arXiv:1101.1147 [gr-qc].

[30] K. A. Malik, JCAP 0511, 005 (2005) astro-ph/0506532.

[31] K. A. Malik, JCAP 0703, 004 (2007) astro-ph/0610864.

[32] D. S. Salopek and J. R. Bond, Phys. Rev. D 42, 3936 (1990).

[33] N. Deruelle and D. Langlois, Phys. Rev. D 52, 2007 (1995) arXiv:gr-qc/9411040.

[34] I. Huston and K. A. Malik, JCAP 0909, 019 (2009) arXiv:0907.2917 [astro-ph.CO]]. 